
allemande

49-1 | 2017

Berlin 1957-1994

\title{
Entre vitrine politique et critique sociétale : Berlin à l'écran des années 1960 à la chute du Mur
}

Diane Barbe

\section{(2) OpenEdition \\ 12 Journals}

Édition électronique

URL : https://journals.openedition.org/allemagne/531

DOI : 10.4000/allemagne.531

ISSN : 2605-7913

Éditeur

Société d'études allemandes

Édition imprimée

Date de publication : 16 juin 2017

Pagination : 133-145

ISSN : 0035-0974

\section{Référence électronique}

Diane Barbe, «Entre vitrine politique et critique sociétale : Berlin à l'écran des années 1960 à la chute du Mur », Revue d'Allemagne et des pays de langue allemande [En ligne], 49-1 | 2017, mis en ligne le 16 juin 2018, consulté le 07 juin 2022. URL : http://journals.openedition.org/allemagne/531 ; DOI :

https://doi.org/10.4000/allemagne.531 


\section{Entre vitrine politique et critique sociétale: Berlin à l'écran des années 1960 à la chute du Mur}

\section{- Diane Barbe*}

C'est en centaines que se comptent les productions filmées «à» et «sur» Berlin, durant la période de la division de la ville: fictions et documentaires, courts et longs métrages, vidéos, bandes d'actualités sont autant de récits et d'images traversés d'éléments communs participant de son identité. Jusqu'en 1989, la «ville siamoise», telle que la nomme Peter Schneider dans Le Sauteur de $M u r^{(1)}$, a occupé les écrans enrichissant deux systèmes de représentation de l'espace urbain, nourris d'éléments propres à chaque partie de la ville et de formes esthétiques spécifiques. Ces deux imageries ont produit un discours, à l'Est et à l'Ouest, participant à la fabrication identitaire de ces deux territoires. Dans une démarche comparatiste de cette filmographie urbaine double, ces images cinématographiques peuvent être observées en ce qu'elles permettent d'enrichir une pensée de l'espace berlinois, une pensée déployée dans le cadre de l'écran. En arpentant le système spatial tel qu'il nous est donné à voir par les caméras, il s'agit de "comprendre-avec-le-cinéma » ${ }^{(2)}$ la ville, afin de saisir dans toutes ses complexités cet objet kaléidoscopique. Cela signifie adopter une démarche géocentrée $^{(3)}$, c'est-à-dire donner la primeur à l'espace et s'attacher à l'analyse du territoire tel qu'il est composé dans les productions cinématographiques.

Des années 1960 à 1989, les images filmées berlinoises expriment, de part et d'autre du Mur, un propos sur la ville qui connaît deux grandes orientations: il participe

* Docteur en études cinématographiques, Université Sorbonne Nouvelle-Paris 3.

1 Peter Schneider, Le sauteur de Mur (1982), Paris, Éditions Grasset, 2000.

2 Antoine Gaudin, L'espace cinématographique. Esthétique et dramaturgie, Paris, Armand Colin, 2015, p. 207.

3 Il s'agit de placer le lieu au cœur de l'attention et de faire d'un référent spatial l'égide sous lequel sont placées les productions cinématographiques soumises à l'analyse. Sur le sujet, voir Bertrand WESTPHAL (dir.), La géocritique mode d'emploi, Limoges, Presses universitaires de Limoges, 2000; Bertrand Westphal, La géocritique. Réel, fiction, espace, Paris, Les Éditions de Minuit, 2007. 
tantôt à faire de Berlin une vitrine politique, il en fait tantôt l'espace d'une critique sociétale. Le présent article entend ainsi questionner la manière dont ces deux discours s'expriment au niveau audiovisuel dans le cinéma de fiction est- et ouest-allemand sur la période retenue ${ }^{(4)}$. Point de repère dans l'expérience de l'espace et dans la pratique du bâti, assignant une charge affective et symbolique aux morphologies urbaines, l'architecture apparaît comme un élément d'analyse particulièrement riche quant à la fabrication d'espaces de significations au sein de la ville. Ainsi, ce sont les images cinématographiques de constructions architecturales emblématiques qui vont retenir notre attention : les héritages passés et les édifices récemment sortis de terre, les lieux emblématiques anciens et modernes d'un Berlin connu et attendu.

\section{Les odes urbaines des années 1960}

Dans les années 1960, les architectures emblématiques sont portées à l'écran dans des odes urbaines où la modernité est vantée, tant à l'Est qu'à l'Ouest, par la composition d'un espace urbain filmé louant la réussite de leur système respectif tant sur le plan urbanistique que sociétal. Des longs métrages tels que Die endlose Nacht de Will Tremper (1963, RFA), Es d'Ulrich Schamoni (1966, RFA), Verliebt und vorbestraft (Erwin Stranka, 1963, RDA) ou encore Der tapfere Schulschwänzer (Winfried Junge, 1967, RDA) donnent à voir Berlin telle une ville-vitrine.

Cela est particulièrement flagrant dans Playgirl, Berlin ist eine Sünde wert (Will Tremper, 1966, RFA) où tout se présente comme apparemment moderne: l'héroïne, Alexandra, une mannequin célèbre et courtisée, voit se succéder séances de photographies et rendez-vous galants. Les lieux qu'elle fréquente, ses relations avec les hommes et sa répartie façonnent un personnage émancipé. "Étrange figure oscillant entre le cinéma commercial et le cinéma d'avant-garde» ${ }^{(5)}$, Will Tremper réalise un long métrage un peu hybride où se côtoient des scènes à l'esthétique ambitieuse et d'autres saturées de clichés et d'invraisemblances du scénario.

Plusieurs séquences dans l'espace urbain viennent ponctuer le récit et l'évolution des flirts de l'héroïne, dans une mise en cadre révélant la modernité de la ville. Le système spatial filmé opère comme une mise en vitrine de Berlin-Ouest où se marient le luxe des villas de Dahlem, l'élégance moderne de nouvelles constructions, le chic des restaurants et les vitrines illuminant la nuit du Kurfürstendamm. Un plan de la Funkturm amorce ce portrait urbain, opérant tel un rappel de la métropole berlinoise des Goldene Zwanziger avec toute la portée symbolique de la référence ${ }^{(6)}$. Ce travelling sur la tour de la radio, devenue un emblème audiovisuel, inscrit topographiquement l'arrivée dans la ville de la protagoniste, ce que son exclamation «Berlin» vient renforcer. Se succèdent par la suite différentes scènes venant en surenchère asseoir la modernité urbaine.

4 Cet article découle de l'étude empirique de près de quatre-vingts longs métrages de fiction est- et ouest-allemands, menée dans le cadre d'une recherche doctorale. Voir Diane BARBE, Berlin(s) à l'écran de 1961 à 1989. Essai de topographie cinématographique, thèse de doctorat d'Études cinématographiques et audiovisuelles, dirigée par Laurent Véray, soutenue le 12 décembre 2016 à l'Université Sorbonne Nouvelle-Paris 3.

5 Ulrich Gregor, «Le Jeune cinéma allemand», Cinéma, nº 110 (1966), p. 36.

6 La tour commença à diffuser des émissions radiophoniques en 1925 et émit le premier programme télévisé régulier au monde en 1935. 
Alexandra est ravie devant la Gedächtniskirche; sur le Kurfürstendamm éclairé dans la nuit, elle va au théâtre et au restaurant. Elle participe à une séance de photographie sur la Ernst-Reuter-Platz, récemment reconstruite, et danse sur le parterre de la Philharmonie, édifiée d'après les plans de Hans Scharoun sur le Kulturforum et inaugurée en 1963. La succession de ces scènes propose une représentation de l'espace urbain édulcorée, collant parfaitement à l'image-vitrine de la ville composée dans ces annéeslà : un Berlin moderne et cosmopolite où il fait bon vivre.

La manière dont le Mur est montré, à deux reprises, appuie ce constat. Lors d'une scène où la mannequin pose devant la paroi de parpaings pour une séance de clichés de mode, son photographe est apostrophé par deux passants, qui lui demandent s'il n'a pas honte de photographier des femmes nues devant le Mur; la protagoniste leur répond « Ne frimez pas trop avec votre Mur!». Cette scène est intéressante parce qu'elle assoie déjà une image du Mur occultant quelque peu sa caractéristique fondamentale de frontière. Le comportement et les propos du photographe et de la mannequin tendent à banaliser le décor, les barbelés et le passage d'une jeep, et par extension le contexte de la Guerre froide.

La frontière est encore suggérée, alors qu'Alexandra traverse la Friedrichstraße, à proximité du Checkpoint Charlie, et qu'elle entend les énoncés diffusés par les hautparleurs du poste-frontière dénonçant le Mur de la honte. Succède à cela une scène, où la jeune femme attend Joachim Steigenwald, promoteur immobilier, pour visiter le chantier de la tour de bureaux, presque terminée, du siège berlinois des éditions Axel Springer. Le parcours ainsi donné à voir entre ces deux lieux use de l'ellipse grâce à la médiation du son: il exprime la proximité du plus célèbre checkpoint de la ville avec le futur bâtiment du fameux groupe de presse, cet immeuble vitrine du capitalisme triomphant.

Un motif récurrent de la mise en images de la ville dans ces années-là est, de surcroît, convoqué dans ces plans: celui du chantier. Les deux filmographies ont, en effet, en partage de porter à l'écran le chantier comme témoin de la modernisation de la ville et de ses transformations. Il se rencontre à plusieurs reprises dans un portrait de Berlin-Est à la modernité affichée: Ein Lord am Alexanderplatz (Günter Reisch, 1967, RDA). Le scénario, drôle et léger, écrit par Kurt Belicke, se fait presque prétexte à la mise en image de l'espace urbain tant la ville s'invite dans ce film comme une protagoniste à part entière. Le héros, Ewald Honig, fraîchement arrivé, sillonne la ville au volant d'une Mercedes-Benz 220 aussi rutilante et tape-à-l'œil que son propriétaire aux manières aristocratiques et à la galanterie excessive. Façade de son prétendu confort financier basé sur une succession de mensonges, la voiture est à l'image de la vie qu'il veut reconstruire à Berlin.

Le système spatial filmé connaît ce qui apparaît comme une progression. Ce qui défile pendant le générique de début en surimpression et la séquence suivante se fait programmatique des évolutions de l'espace urbain telles qu'elles vont être portées à l'écran. Pendant près de deux minutes, plans fixes sur certaines architectures alternent avec des travellings et des panoramiques suivant la Mercedes ou filmés depuis son habitacle. Le centre-ville historique défile au rythme de la conduite d'Ewald chantonnant et ces vues sont entrecoupées de plans fixes d'immeubles dynamités et de façades s'effondrant dans un grand fracas. Le Rotes Rathaus, l'allée Unter den Linden et la porte de Brandebourg, le Deutsche Oper laissent place aux nouvelles constructions d'après-guerre: au patrimoine architectural de la Museumsinsel, affichant une légitimité territoriale, s'adjoignent les nouvelles constructions de l'Alexanderplatz, fleurons 
des politiques de rénovation de cette partie de la ville. Les plans fixes d'immeubles dynamités viennent asseoir visuellement la construction d'un centre de Berlin-Est moderne, qui se fait de plus en plus présent à l'écran à mesure que l'on avance dans le film. Ces vues de destruction d'anciens immeubles se présentent comme des combinaisons audiovisuelles éloquentes et puissantes, significatives de la logique de transformation de la ville en vue de sa modernisation: le réel inhérent au film, en renvoyant par un jeu de référentialité à l'extrafilmique, invite le spectateur à « retrouver une expérience visuelle dans une image répétitive, condensée, maîtrisable ${ }^{(7)}$. L'effondrement de ces Altbauten renverse la figure du déchet, qui, dans son rapport à la temporalité, évoque l'état antérieur et dépassé d'une chose, d'un système. Dans cet espace urbain est-berlinois en mutation, l'image du déchet urbain suite à une destruction revêt une connotation positive: il ne s'agit plus des tonnes d'éboulis de la ville bombardée en 1945 mais d'un dynamitage décidé pour la métamorphose moderne de la ville. À la catastrophe d'hier répond le progrès d'aujourd'hui ${ }^{(8)}$.

Si la première moitié du long métrage est ainsi ponctuée de vues et de scènes se déroulant dans le quartier de Mitte dans une forme d'affichage du classicisme de ses lieux emblématiques, dans la seconde moitié du film, place est faite aux monumentalités modernes autour de l'Alexanderplatz, telle la Haus des Lehrers, la Kongresshalle et la Karl-Marx Allee. Le mouvement vers l'est que donne à voir la progression du système spatial filmé traduit le cheminement vers la modernité, dans un subtil mariage entre passé et présent, où ce dernier prend l'ascendant comme en témoigne une des dernières scènes urbaines du long métrage. Deux panoramiques de grand ensemble embrassent la ville, l'œil de la caméra tourné vers l'Est. La ville s'offre au regard, parée de ses nouveaux immeubles quadrillant l'espace; la Haus des Lehrers et ses fresques en mosaïque, aisément reconnaissables, rappellent la modernité architecturale incarnée notamment dans la rénovation de l'Alexanderplatz et les réalisations du célèbre architecte Hermann Henselmann. L'image de l'appareil de levage s'imposant dans le champ figure, de surcroît, en surenchère la reconstruction du centre de Berlin-Est, tel le symbole du chantier de modernisation de l'espace urbain. Ces prises de vue, cadrées en plan d'ensemble, et les nombreuses architectures emblématiques portées à l'écran viennent asseoir topographiquement la réplique d'un des personnages du récit, la criminologue Johanna Farkas, à son arrivée à la Ostbahnhof: «Das ist unsere Hauptstadt der $D D R »$. Il est intéressant me semble-t-il d'y voir la manifestation d'une efficience du regard - à travers l'œil de la caméra en surplomb - au service de la ville-vitrine.

Ce regard éminent et le symbole qu'il charrie, associés à ces vues de très grand ensemble, se rencontrent aisément dans les films est-allemands des années 1960. La volonté d'afficher la modernité du nouveau centre de Berlin-Est transparaît par exemple dans les vues en plongée depuis des immeubles en construction au sud de l'Alexanderplatz dans Verliebt und vorbestraft (Erwin Stranka, 1963, RDA) ${ }^{(9)}$. Der

7 Jacques Aumont, L'image, Paris, Nathan, 1990, p. 59.

8 Il est à noter que ces plans se rencontrent tout au long des années 1970 dans la cinématographie estallemande, notamment dans l'un des plus grands succès des studios de la DEFA Die Legende von Paul und Paula (Heiner Carow, 1973, RDA), et même jusqu'au début de la décennie qui suit tel qu'en témoigne Sabine Kleist, 7 Jahre (Helmut Dziuba, 1982, RDA).

9 Sur ce sujet, voir Diane BARBE, «Ville nouvelle à l'écran: le stéréotype filmé des grands ensembles berlinois (1961-1989)», Revue Trait d'Union (en ligne), nº 7 (2017), à paraître. 
tapfere Schulschwänzer (Winfried Junge, 1967, RDA), encore, propose pour faire suite au générique des plans d'ensemble de lieux emblématiques de Berlin-Est montés en alternance avec des instantanés du jeune protagoniste, Thomas. Ces vues, à l'instar de celles qui ponctuent fréquemment la diégèse, donnent à voir la Museumsinsel, le Rotes Rathaus, le Gendarmenmarkt (à l'époque de la RDA, Platz der Akademie), le Berliner Dom, la Marienkirche ou encore le café Moskau sur la Karl-Marx Allee. Elles obéissent à un cadrage faisant que la partie occidentale de la ville n'est à aucun moment ni montrée, ni suggérée. Le «Das ist Berlin», prononcé en voix off sur un plan du cœur historique, associe, dans une forme de surenchère, le nom de la ville à la succession d'images apparaissant à l'écran. Dans ce film dont le scénario est prétexte à une succession de scènes opérant comme un album de cartes postales animées participant à une représentation urbaine telle une vitrine, ces plans insérés au cœur même du récit peuvent se lire comme des «manifestations d'un regard panoptique ${ }^{(10)}$, où la caméra recouvrerait la toute puissance fantasmatique de l'œil. Ce regard total portant une ambition globalisante, embrasse, en récurrence, le cœur, l'intérieur de la partie orientale de la ville. Il l'unifie autour de son centre historique et semble quelque part se faire l'instrument d'une possible maîtrise du monde urbain en affirmant, par la répétition, l'identité de la ville. L'angle de prise de vue en plongée ou bien la position de la caméra en surplomb confortent une vision hégémonique sur l'espace montré que le travail sur la lumière, dense et chaude, souligne d'un vernis flatteur. Ces plans d'ensemble ont de surcroît en commun le fait d'être filmés en caméra objective : ce sont des vignettes jalonnant la diégèse, des «cartes postales animées » marquant des pauses dans le récit au service d'une mise en valeur de la ville-personnage. Ces vues ont ainsi la particularité d'être déconnectées d'un possible regard des protagonistes, de n'y faire aucune référence explicite, accentuant, de fait, une impression de globalité. La caméra y symbolise un œil collectif, incarnation de la masse et de la communauté.

Ces mises en cadre de certains lieux et motifs qui se succèdent sont au service de ces odes à la modernité qui vantent la ville-vitrine dans les années 1960. Moderne et charmant, ce paysage se nuance peu à peu et les protagonistes l'occupent de manière plus contrastée à partir du début de la décennie qui suit.

\section{L'image carte-postale de la ville: entre complexification et retournement dans les années 1970}

Les années 1970 amorcent ainsi une complexification du système spatial filmé où les lieux emblématiques se chargent d'une portée sémantique par l'expérience des personnages du récit, qui alourdit d'un signifié l'image carte-postale de la ville. Les deux Berlin ont dès lors en partage de se déployer à l'écran de façon kaléidoscopique. Certains longs métrages composent un espace urbain filmé affichant une forme de neutralité, tandis que d'autres s'emparent du paysage pour faire un territoire où s'exprime un propos critique.

Dans la filmographie est-allemande, la vision panoptique circonscrivant Berlin à sa seule partie orientale, observée quelques années plus tôt, laisse place à un usage des

10 Patrick Vienne, "You are here: la ville en plan d'ensemble dans le cinéma américain contemporain », in: Julie Barillet, Françoise Heitz, Patrick Louguet, Patrick Vienne (dir.), La ville au cinéma, Arras, Artois Presses Université, 2005. p. 56. 
plans de très grand ensemble et en plongée permettant d'embrasser la ville et de donner à voir l'autre côté. Un long métrage est, à ce titre, éloquent. Hostess (Rolf Römer, 1976, RDA) dresse le portrait de Jette, hôtesse à la tour de la Télévision animant visites guidées de monuments et tours dans la ville en bus. Berlin-Est y est révélé comme une ville touristique, où le montage, telle une brochure touristique, décline les différents lieux incontournables de la ville. La similitude des vues urbaines dans cette séquence avec les photographies qui ont pu être observées dans les fascicules à l'intention des visiteurs $^{(11)}$ est frappante et conduit à considérer ces mises en cadre comme un album de cartes postales animées emblématiques de la partie orientale de Berlin.

Que le système spatial filmé dans Hostess réponde de l'image-vitrine est indéniable. Toutefois, trois courtes scènes, portant à l'écran la porte de Brandebourg, invitent à complexifier l'analyse de l'espace urbain filmé dans ce long métrage. La première se déroule dans la salle panoramique, en haut de la tour de la Télévision, où la protagoniste commente en anglais une visite de ce Berlin vu d'en haut. Alors qu'elle explique que cette partie de la ville fut détruite à près de $80 \%$ lors de la Seconde Guerre mondiale, la caméra s'attarde sur ce qu'elle nomme "the center of the town before the war»: une partie de l'Île des Musées avec le Berliner Dom, Unter den Linden et ses bâtiments célèbres et, à son extrémité, la porte de Brandebourg ouvrant sur la Straße des 17. Juni. Le travelling optique dure cinq secondes et se rapproche de la porte jusqu'à la distinguer nettement et montrer l'absence d'activité autour d'elle. Ce vide "humain» matérialise l'existence du Mur, qui n'est pas visible à l'écran, et semble venir nuancer le tableau que dresse la guide des reconstructions et des rénovations entreprises par les autorités est-allemandes pour faire de Berlin-Est la façade de la République démocratique. Une autre image de la porte de Brandebourg survient lors d'une visite guidée à travers la ville à bord d'un bus. Ce travelling de trois secondes fait suite à une prise de vue de la fontaine Schwebender Ring sur la Karl-Marx Allee et précède un plan du Dom et d'Unter den Linden. La scène finale, enfin, peut être lue comme un prolongement d'un procédé centrifuge de mise en cadre de l'espace urbain où il ne s'agit plus d'aller du centre vers la périphérie mais d'embrasser la ville dans sa totalité. Plusieurs travellings optiques arrière partant d'un plan rapproché sur le dernier étage d'un immeuble, puis s'éloignant pour inclure les toits alentour, sont suivis de vue aériennes révélant Berlin. L'apparition de l'église du Souvenir et de la colonne de la Victoire, incarnant la partie occidentale de la ville, vient en outre rappeler que si Berlin-Est est un objet unifié, identifié et identifiable comme un espace urbain complexe, il existe un autre espace urbain au-delà du Mur. La porte de Brandebourg est ainsi insérée dans cet «album filmé» de Berlin-Est et révélée comme intégrée à la topographie berlinoise en tant que monument historique ayant un attrait touristique. Toutefois, la charge mémorielle et symbolique en référence à la frontière n'en est pas moins gommée. Ce qui apparaît dans les images filmées de la porte de Brandebourg témoigne d'un paradoxe dans les expressions complexes de la représentation du Mur en RDA. Le photographier ou le filmer signifiait prendre le risque de menaces et de

11 À titre d'exemples, voir: Berlin, capitale de la République démocratique allemande, Dresde, Verlag Zeit im Bild, 1987; Berlin Hauptstadt der DDR (1977), Berlin, VEB Tourist Verlag, 1983; Berlin. La capitale de la RDA salue ses visiteurs, Berlin-Est, Berlin-Information, 1984; Kurzgefaßt: Berlin, Berlin, Presse- und Informationsamt des Landes Berlin, 1966; Eckart Stratenschulte, Berlin-Est, Berlin, Informationszentrum, 1988. 
répression ${ }^{(12)}$. Exceptés les Mauerfilme $e^{(13)}$ et l'iconographie des premières années de son existence, l'enceinte bétonnée n'était jamais représentée comme telle et rapidement, la porte de Brandebourg devint le lieu incarnant la frontière dans les dessins et les clichés. Pour autant, il était possible de faire du monument des «prise[s] de vue d'un point de vue touristique en conformité avec la doctrine socialiste» ${ }^{(14)}$.

À l'instar de Rolf Römer, Rosa von Praunheim, dans Berliner Bettwurst (1973, RFA), usant d'une mise en cadre de l'espace urbain tel un album de cartes postales animées, propose un collage de motifs intrinsèques à l'image-vitrine de Berlin-Ouest en les détournant systématiquement par le jeu des protagonistes ou bien en les juxtaposant. Ces différents motifs, de surcroît, se superposent à l'écran, adjoignant à la prise de vue urbaine des objets sonores, des représentations dessinées et des paroles attachées à une vision attendue de la ville, où le kitsch se confronte au poids de l'Histoire.

Une séquence de près de trois minutes donne à voir le couple de protagonistes, filmés, en plan fixe, assis au premier étage d'un bus passant devant un certain nombre de lieux emblématiques. S'improvisant guide touristique pour Luzy, Dietmar commente ce qu'ils voient défiler derrière les vitres. S'il n'est pas aisé pour le spectateur, au regard de l'angle de prise de vue, de distinguer nettement les lieux mentionnés, la ville défile, en réalité, à travers les propos du couple et les toponymes cités, dans une forme de visite aveugle de la ville-vitrine. Il évoque la Siegessäule, son déplacement en 1938 sur la Straße des 17. Juni. En répétant à l'envi combien l'endroit est célèbre, Dietmar pointe du doigt l'Europa-Center, l'Église du Souvenir, le siège des éditions Alex Springer, le Checkpoint Charlie sur lequel il s'attarde dans ses explications. Grossissant le trait du registre comique en jouant avec la caricature, le didactisme excessif, presque artificiel, de Dietmar, usant abondamment de la répétition, ses gestes très maniérés, associés à la légèreté de Luzy oscillant entre indifférence et excentricité, enveloppent la séquence d'un ton flirtant avec le burlesque.

S’ensuit une scène au pied du Mur, où le bus s'est arrêté devant la façade d'un Altbau de la Bernauer Straße, dont les fenêtres et portes ont été murées parce que le bâtiment se trouvait sur la ligne de démarcation. Dietmar relate à Luzy l'histoire récente de la célèbre rue et, de fait, celle de la frontière urbaine. Les deux personnages sont filmés devant une croix de bois ornée d'une couronne et d'un bouquet de fleurs, placée là en hommage à une des victimes du Mur ayant trouvé la mort lors d'une tentative de fuite vers Berlin-Ouest ${ }^{(15)}$. Suite à leur dialogue a lieu une séance de photographies où, avec

12 Sur les photographies clandestines de la frontière, voir Berliner Mauer: Fotos verboten! Die heimlichen Fotos von Detlef Matthes in Ost-Berlin, Berlin, BStU Bildungszentrum, 2013.

13 Sur les Mauerfilme, voir Cyril BuffeT, "RDA - MON AMOUR". La représentation du mur de Berlin dans le cinéma est-allemand», Allemagne d'aujourd'hui, n 189 (2009), p. 190-204; Diane BARBE, «Projecteurs sur le Mur. La représentation du Mur de Berlin dans le cinéma de fiction est- et ouestallemand de 1961 à 1990 », in: Christin Niemeyer, Ulrich Pfeil (dir.), Der deutsche Film im Kalter Krieg. Cinéma allemand et Guerre froide, Bruxelles, Peter Lang, p. 197-212.

14 Jean-Louis Georget, «Photographier le Mur», in: Nicole Colin, Corine Defrance, Ulrich Pfeil, Joachim Umlauf (dir.), Le Mur de Berlin. Histoire, mémoires, représentations, Bruxelles, Peter Lang, 2016, p. 238.

15 Une première croix avait été placée à l'endroit où Ida Siekmann se blessa mortellement le 22 août 1961 , en sautant du troisième étage de l'immeuble sis au 48 de la Bernauer Straße, dont la porte avait été murée le matin même. Elle est considérée comme la première victime du Mur. D’autres croix furent par la suite installées en mémoire d'autres personnes décédées lors de tentatives de fuite. 
un enthousiasme exubérant, ils prennent la pose devant l'objet mémoriel au premier plan, la frontière au second. Le comportement du couple répond de la mise en scène induite par la présence double d'un objectif photographique et d'un lieu emblématique, touristique, objet d'un parcours menant jusqu'à lui. Aucune différence n'est faite entre ce lieu-là, le Mur, et n'importe quel autre: leurs attitudes, gestes et expressions sont similaires à ceux vus dans d'autres scènes, pour d'autres photographies dans la ville. En gommant les spécificités du lieu et en bousculant l'attitude attendue des touristes en visite, Rosa von Praunheim impose le décalage. Décalage chromatique dans les teintes criardes de leurs vêtements tranchant sur les dégradés de gris et de marron du bâti environnant. Décalage de rythme aussi lorsque le couple s'élance en courant vers la plateforme d'observation en se tenant la main, tandis que les autres touristes sont discrets, observateurs concentrés, conscientisant l'expérience géographique de la Guerre froide. Un décalage enfin entre la charge mémorielle incarnée dans la croix et leur enthousiasme exagéré, la légèreté de ton de leurs propos et de leurs réactions. S’agitant, littéralement, devant l'objet commémoratif, Luzy exprime sa déception de ne pas voir «les barbelés dont on lui a parlé» avant de convenir avec Dietmar de l'urgence de prendre des photos au vu de la courte durée de la pause du bus. Dans cette scène flirtant avec l'absurde, le comique improbable de la situation tourne en dérision ce tourisme du Mur et la valorisation de la frontière comme lieu touristique dans l'aménagement du territoire urbain. La présence de la croix, rappelant les politiques commémoratives de ses victimes, invite à s'interroger aussi sur le détournement de ce symbole, non pas au regard de la mémoire de ces individus, mais plus en ce qu'il participait à la charge emblématique de la Bernauer Straße. Dès 1961, du fait de sa position, la rue se fit icône médiatique et elle le resta en devenant un des lieux incontournables des circuits touristiques et des visites officielles.

Le détournement de l'image-vitrine de Berlin-Ouest trouve un prolongement dans un plan fixe, à la vingtième minute du long métrage, portant à l'écran un coussin, en satin bleu nuit, orné de broderies représentant, à ses quatre coins, la Funkturm, le grand magasin KaDeWe, le mémorial pour le Pont aérien situé devant l'aéroport de Tempelhof et l'Église du Souvenir. La porte de Brandebourg figure au centre, audessus du nom de la ville écrit en lettres capitales et de l'ours, son emblème, ceint d'un écusson. Cette composition kitsch brodée d'image de lieux emblématiques opère comme un finale du portrait touristique de la ville avant d'ouvrir sur une appréhension plus hétéroclite de l'espace urbain filmé. Avec une dynamique centrifuge, le système spatial donné à voir dans ce long métrage semble être construit en deux parties où l'image de ce coussin opère telle une césure entre un premier temps, s'emparant pour la détourner de l'image-vitrine de Berlin-Ouest, et un second apparaissant comme un collage de motifs urbains qui ne sont plus touristiques, entrecoupés de longues scènes en intérieur et d'un voyage du couple à Majorque. Plus aucune prise de vue de lieux célèbres n'apparaît après ce plan du coussin, sorte de condensé iconique et symbolique de Berlin-Ouest. Sont réunis des monuments emblématiques ayant une charge sémiotique et historique forte renvoyant au passé d'avant-guerre (excepté le mémorial pour le Pont aérien) tout en symbolisant l'expérience de la Guerre froide. La porte de Brandebourg et sa position centrale dans la composition, particulièrement, mettent en exergue le palimpseste berlinois. Concentrant les strates de passé, investie par les gouvernements successifs, depuis la fin du XIX ${ }^{\text {e }}$ siècle, d'un message 
politique $^{(16)}$, elle raconte la constitution de l'État-nation en 1871, la surenchère de la propagande nazie et l'exaltation du sentiment national, puis la «catastrophe allemande» en 1945. Avec la partition du pays, elle s'est retrouvée à l'extrémité de la zone soviétique puis de la partie orientale de Berlin, et devint, après la construction du Mur, un lieu inaccessible, au milieu du no man's land, symbole de la division de la ville. Et, de fait, ce lieu de mémoire par excellence de l'histoire allemande récente était le seul monument commun aux deux filmographies ${ }^{(17)}$, qui, tout en incarnant la frontière, est devenu un motif iconique objet d'enjeux mémoriels et politiques propres à chaque système de représentation.

Ces broderies sur satin, de surcroît, rapprochent ce coussin des babioles kitsch, ces petits objets vendus dans des kiosques pour touristes, dont la surcharge, les matériaux bon marché et la fonctionnalité incertaine font qu'ils sont bien souvent taxés de «mauvais goût». Détournés, les lieux emblématiques ainsi présentés sont, dans cette mise en abîme figurée, non pas seulement exposés mais presque caricaturés, artificialisés, invitant à jouer avec les mots, à rapprocher ce brocart de soie, parce que flirtant avec le sarcasme, avec son homonyme, le brocard, la raillerie.

Hostess et Berliner Bettwurst partagent une mise en cadre de l'espace urbain composite. Les architectures emblématiques telles qu'elles ont été observées apparaissent à l'écran comme des motifs urbains notoires, admis et connus comme tels, dans une forme de mise en vitrine qui, jouant de certains clichés, correspond à une représentation "attendue», tout en la complexifiant par l'appréhension de l'espace des protagonistes. Elle n'est plus seulement le support d'un propos topo-centré ouvrant une réflexion sur la "ville carte postale», elle s'alourdit, elle s'épaissit d'un signifié relevant de l'expérience, celle des personnages dans le réel diégétique.

\section{L'altération de l'image-vitrine: la ville, espace d'une critique sociétale}

À Berlin-Ouest, l'éloignement d'avec la ville-vitrine, marqué dès le début des années 1970 et s'affirmant au milieu de la décennie, se traduit par des contre-images, inscrites dans une tradition contre-culturelle que la fin des années 1960 a profondément ancrée dans cet espace isolé( ${ }^{(18)}$. Elles expriment une critique du système dominant et de son modèle économique par la territorialisation d'un propos politique s'attachant aux architectures et à la frontière. La modernité s'incarne dans l'étoile Mercedes brillant dans la nuit de l'Europa Center tandis que les monuments historiques sont de près ou de loin liés à la dictature fasciste, réactivant dans le présent un passé lourd. L'histoire se déploie dans l'espace urbain, pesant sur son appréhension, sur son appropriation. La "ville-Phoenix», témoin de sa capacité à se relever du chaos de l'après-1945, est délaissée pour la ville-mémoire. Helke Sander (Redupers, 1978, RFA), Ulrike Ottinger

16 Sur le sujet, voir Stéphane FüZESSERY, «L'emblème et le symbole. Un siècle d’imageries cinématographiques de la porte de Brandebourg», Vingtième Siècle. Revue d'histoire, n 72, oct.-déc. 2001, p. 89-96; Étienne François, Hagen Schulze (dir.), Mémoires allemandes, Paris, Gallimard, 2007.

17 Voir précédemment les remarques sur Hostess (Rolf Römer, 1976, RDA).

18 Sur le sujet, voir, entre autres, Charlotte Вомy, André Combes, Hilda Inderwildi, Contre-cultures à Berlin de 1960 à nos jours, Cahiers d'Études germaniques, n 64 (2013); Nicole Colın, "Splendid isolation. Biotopes créatifs à l'ombre du Mur», in: Colin/Defrance/Pfeil/Umlauf (dir.), Le Mur de Berlin (note 14), p. 197-212. 
(Bildnis einer Trinkerin, 1979, RFA), Rainer Werner Fassbinder (Die dritte Generation, 1979, RFA), Helma Sanders-Brahms (Die Berührte, 1981, RFA) ou Thomas Brasch (Domino, 1982, RFA) écrivent en images filmées un espace urbain tel un feuilleté de mémoire, un sismographe de l'histoire que le présent ne parvient pas à apaiser.

Le Reichstag et la Colonne de la victoire (Siegessäule) sont deux monuments dont les expressions cinématographiques sont caractéristiques de l'expression d'un "passé qui ne passe pas». Dans Bildnis einer Trinkerin (Ulrike Ottinger, 1979, RFA), par exemple, l'image de la Siegessäule est éloquente. La Straße des 17. Juni, sur laquelle se dresse le monument, se fait le décor du passage de la protagoniste, lors d'un de ses périples alcoolisés, suivie par son acolyte, la «clocharde du Zoo». La scène est précédée d’une de leurs dérives nocturnes les ayant menées en bordure du Mur, s'achevant sur un plan fixe où, longeant la frontière, elles disparaissent dans la nuit noire au fond du cadre, les pas incertains de l'une répondant au roulis du chariot de l'autre, l'éclairage des lampadaires composant une image en pointillés. Devant la caméra d'Ulrike Ottinger, le Mur trouve sa place dans le système spatial filmé berlinois et cette présence dans les images de la ville tend à s'insérer dans une réflexion plus large sur les notions d'enfermement, de frontière - matérielle ou non - entre les individus, les groupes sociaux, les parcours de vie. Le plan suivant, filmé en légère contre-plongée au niveau du sol, voit leurs jambes entrer dans le champ; l'héroïne, d'une démarche tout aussi distinguée, altière dans sa robe de soirée, qu'incertaine, sous l'effet de l'alcool, se dirige lentement vers la Siegessäule. Elle choisit ce lieu, cette rue, pour jeter son sac à main dont le contenu se répand sur la chaussée, avant d'être ramassé par sa comparse. Un homme se saisit aussi d'une trousse, tente d'approcher l'héroïne pour la lui rendre, renonce devant son indifférence et se dirige vers le trottoir où il s'assoit, déposant sur le bitume les menus effets qu'elle renfermait, avant de les photographier un à un. Leurs clichés se succèdent à l'écran conférant à leur banalité une valeur quasi documentaire oscillant entre l'archive et la trace. Il est tentant de lire cet enchaînement de plans au prisme de l'identité : devant la mémoire de la nation incarnée dans la colonne, la protagoniste, en abandonnant son sac, semble se défaire de sa propre identité, inscrite sur les papiers officiels que ce dernier renferme le plus souvent, mais aussi d'une partie d'intimité, signifiée par ces objets a priori banals et pourtant personnels contenus dans la pochette. Il y aurait presque, face à l'histoire collective, une négation de la sienne propre, qui trouverait un prolongement dans son alcoolisme et la fuite dans les paradis artificiels.

C'est encore la charge historique du monument dont s'empare Thomas Brasch dans Domino (1982, RFA). Portant à l'écran les déambulations et les rencontres d'une jeune actrice, Lisa, c'est un Berlin hivernal et enneigé qui y est portraituré comme un lieu épais de l'Histoire, où le passé vient permuter le présent inlassablement.

Ce Berlin au passé dans le présent transparaît dans la mise en cadre de certaines architectures et notamment celle de la colonne de la Victoire et du Reichstag. En moto, la protagoniste et un ami traversent la nuit berlinoise, roulant sur la Straße des 17. Juni jusqu'à la Siegessäule dont ils font le tour. L'emblème de la victoire de la Prusse en 1871 et de l'unification du Kaiserreich semble refléter ici la vision de Walter Benjamin, qui écrira, lors de son exil en France, combien ce monument, symbole du patriotisme allemand, risque de n'être plus qu'un tombeau de l'Histoire, incarnation des angoisses dans le contexte de résurgence du nationalisme fasciste des années 1930. 
«Dans la nuit glaciale, la statue impose une présence lugubre et écrasante. Dans la rude topographie du Berlin de la Guerre froide, «Siegesallee», le boulevard qui mène au monument, est tronqué par le Mur de Berlin, les personnages en étant réduits à encercler le lieu symbolique en signe de stagnation ${ }^{(19)}$.

La présence dans le champ de la Siegessäule, tandis que les deux personnages se rendent chez Lisa pour y passer la nuit, semble annoncer le silence et la lenteur des plans qui vont suivre. Elle se rapproche des déambulations de Lisa, la nuit de la SaintSylvestre: sur le parvis du Reichstag, elle fait la rencontre d'une jeune fille avec qui elle troque son manteau contre un chapeau; cette dernière s'exclame en voyant Lisa: «Maintenant tu as l'air de quelqu'un qui partirait à la guerre». Ces deux scènes façonnent un espace où les cauchemars de Lisa, ses angoisses, prennent corps peu à peu jusqu'à cette séquence fantasmagorique où les feux d'artifice, illuminant le ciel berlinois le $1^{\text {er }}$ janvier, semblent être des grenades et des bombes explosant. La guerre, celle passée, et celle dont on craint le surgissement, dans le contexte de ce début des années 1980, devient un motif de plus en plus présent dans le récit, jusqu'à devenir obsessionnelle, expression des angoisses de la protagoniste.

Derrière la crise existentielle de l'héroïne, Thomas Brasch critique ouvertement les structures politiques et économiques du système capitaliste écrasant l'individu: «les films sont d'abord des images » ${ }^{(20)}$ et celle récurrente du Kurfürstendamm illuminé, occupé par une foule désordonnée qui poursuit le même objectif d'achats de présents pour Noël, exprime les effets aliénants de la société de consommation. Le premier des cauchemars de Lisa porté à l'écran la révèle paniquée, bloquée dans un grand magasin dont elle ne peut ouvrir les portes, littéralement enfermée dans ce «temple» de la consommation et engloutie par ce «paradis» capitaliste. Les trophées et fétiches de la prospérité en République fédérale, décalque de la société américaine, ne parviennent pas à rendre acceptable une économie basée, entre autres, sur la production d'armes ${ }^{(21)}$. Pour ce «cinéaste à l'écart des modes, mais avec un sens du présent et en particulier des décors urbains » ${ }^{(22)}$, la notion de "progrès» est frontalement rejetée, dans la continuité de la pensée benjaminienne. Occupée par un présent qui n’est pas encore là, et un passé qui ressurgit en permanence, Lisa trébuche dans ce Berlin agissant comme un entrepôt collectif de souvenirs et de traumatismes dont les vitrines illuminées du KaDeWe ne sont qu'un masque de paillettes.

Ces deux longs métrages, pris en exemple, témoignent d'une représentation des architectures emblématiques, qui, par la relation espace-personnage, bouleverse la manière dont elle participait à l'image-vitrine de la ville au service d'un discours critique. À partir de la fin des années 1970, à Berlin-Est aussi, un certain nombre de fictions donnent à voir un repli sur le quotidien et sur l'individu qui se spatialise dans les représentations cinématographiques de la ville.

19 Christian Rogowski, “To be continued.” History in Wim Wenders's "Wings of Desire” and Thomas Brasch's "Domino" ", German Studies Review, vol. 15, n 3 (1992), p. 548.

20 Thomas BRAsch, propos recueillis par Jeanne von Stebut, "Cinéastes berlinois», Jeune cinéma, $\mathrm{n}^{\circ} 139(1981 / 82)$, p. 10.

21 En 1980, Thomas Brasch, avec Günter Grass, Peter Schneider et Sara Kirsch, écrivit une lettre ouverte au chancelier Helmut Schmidt lui demandant de résister au déploiement de missiles américains sur le sol allemand, appréhendant la possibilité d'un troisième conflit mondial. 
Lothar Warneke porte ainsi à l'écran dans Die Beunruhigung (1982, RDA) l'Alexanderplatz, fierté de la modernité de la capitale de la RDA, comme l'espace de l'expérience de la solitude. Portrait d'une psychologue, Inge, le film relate la journée précédant son opération pour un cancer du sein, lors de laquelle elle s'engage dans un itinéraire au cœur de la ville ponctué de rencontres et de moments solitaires, structuré par des vues urbaines qui viennent accompagner le nouveau regard qu'elle porte sur son existence suite à ce diagnostic. Son cheminement introspectif la conduit à questionner son présent et à remonter dans son passé, constamment ancrée dans l'espace qu'elle occupe, observant les lieux dans lesquels elle évolue. La ville donne corps à cette expérience réflexive, se calquant sur ses va-et-vient entre passé et présent. Son parcours solitaire est associé au Berlin de la modernité et des plans de l'Alexanderplatz, étrangement vide, scandent son après-midi, tandis que c'est dans des quartiers aux architectures plus anciennes, tel Prenzlauer Berg, qu'elle retrouve amies et vieilles connaissances. Son cheminement invite à une lecture individuelle du système spatial filmé, une appréhension de l'espace au regard de la sphère de l'intime. La ville semble portée à l'écran avec autant de force que de banalité.

Ce long métrage est emblématique d'une évolution dans la composition filmée de Berlin-Est. On observe ainsi, à la charnière des décennies soixante-dix et quatre-vingt, combien les personnages de fiction s'approprient l'espace berlinois en injectant du privé dans le public: ils désaffectent certains lieux emblématiques des années 1960, comme la Karl Marx Allee, et n'occupent pas les espaces investis d'une charge symbolique forte par le régime, tel le Palast der Republik. Plus qu'une rupture, cette délocalisation et l'absence à l'écran de certains lieux expriment une négation de l'ordre spatial prévalant dans l'imagerie officielle. Cette insoumission au cadre d'un discours dominant trouve un prolongement dans la marginalité de certains protagonistes - ou bien est la résultante. La dernière décennie de la période voit les écrans se peupler de personnages entretenant avec leur Berlin respectif des relations flirtant avec la dysphorie. Les sujets sont physiquement dans la ville mais n'en partagent pas les valeurs. La chanteuse de Solo Sunny (Konrad Wolf, 1980), la jeune femme de Bürgschaft für ein Jahr (Hermann Zschoche) ou le protagoniste de Die Architekten (Peter Kahane, 1990), par exemple, voient leur malaise, leur mal-être se déployer dans l'espace. Leurs désillusions s'écrivent dans l'espace urbain, ils sont déchirés entre un ici physique et un ailleurs psychologique. La ville est donnée à voir comme un territoire qui, s'éloignant d'un discours dominant et d'une image officielle, devient oppositionnel et se fait l'espace d'une critique sociétale.

En somme, l'analyse des images cinématographiques de lieux emblématiques berlinois, de part et d'autre du Mur à travers les exemples choisis, révèle, non seulement deux orientations dans les discours attachés à la ville - l'une révélant son image-vitrine, l'autre faisant du territoire l'espace d'une critique sociétale - mais, de plus, une évolution allant de l'une à l'autre. Si dans les années 1960, les portraits berlinois célèbrent la métropole moderne et la réussite de leur système respectif par une mise en cadre des architectures, la décennie qui suit amorce une complexification de ces deux vitrines politiques en détournant notamment l'image touristique de la ville. Dans un mouvement presque synchrone dans les années 1980, un certain nombre de longs métrages de fiction est- et ouest-berlinois composent à l'écran un territoire au service d'une critique de leurs sociétés respectives jusqu'à dépeindre l'échec de leurs systèmes politique 
et économique. Ces conclusions invitent à élargir le champ des motifs urbains analysés et ouvrent des questionnements sur la manière dont les représentations filmées des lieux en "nom commun» que sont les rues, les places, les boulevards et les immeubles d'habitation viennent appuyer ou, a contrario, nuancer cette évolution du propos et de la charge identitaire contenus dans l'image de Berlin au cinéma.

\section{Résumé}

Des années 1960 à 1989, de nombreux films portent à l'écran Berlin-Est et-Ouest, traduisant de part et d'autre du Mur, un propos sur la ville divisée. Le présent article entend questionner, à travers quelques exemples choisis, les expressions audiovisuelles de ce discours urbain dans le cinéma de fiction est- et ouest-allemand sur la période retenue, en s'emparant des images cinématographiques de constructions architecturales emblématiques. L'analyse de ces images révèle deux orientations dans les discours attachés à la ville : l'une révélant son image-vitrine, l'autre faisant du territoire l'espace d'une critique sociétale. Elle traduit aussi une évolution allant de l'une à l'autre. Si dans les années 1960, les portraits berlinois célèbrent la métropole moderne et la réussite de leur système respectif, la décennie qui suit amorce une complexification de ces deux vitrines politiques en détournant notamment l'image touristique de la ville. En un mouvement presque synchrone dans les années 1980, un certain nombre de longs métrages de fiction est- et ouest-berlinois composent à l'écran un territoire au service d'une critique de leurs sociétés respectives.

\section{Zusammenfassung}

Zwischen 1960 und 1989 werden in der Bundesrepublik Deutschland und der DDR zahlreiche Filme gedreht, die in West-oder Ost-Berlin spielen und ein jeweils eigenes Bild der geteilten Stadt entwerfen. Anhand ausgewählter Beispiele aus ost- und westdeutschen Spielfilmen untersucht der vorliegende Beitrag die audiovisuellen Aspekte dieses urbanen Diskurses, wobei der Schwerpunkt auf die Darstellung symbolträchtiger Orte und der dort angesiedelten Architektur gelegt wird. Grundsätzlich lassen sich dabei zwei Perspektiven unterscheiden: eine, welche die Stadt wie ein Schaufenster darstellt, und eine, die den städtischen Raum zur Bühne von Gesellschaftskritik macht. Mittels eines diachronen Vergleichs wird eine Verschiebung beider Perspektiven herausgearbeitet: So feiern die Filme auf beiden Seiten in den 1960er Jahren Berlin als moderne Metropole und betonen dadurch den Erfolg des jeweils eigenen Systems. Im Folgejahrzehnt werden die Stadtdarstellungen komplexer, indem sie beispielsweise das touristische Image der Stadt entstellen. Auf nahezu übereinstimmende Weise machen einige Spielfilme der 1980er Jahre dann den städtischen Raum zum Austragungsort gesellschaftskritischer Betrachtungen. 